\title{
Predicting solvation free energies and thermodynamics in polar solvents and mixtures using a solvation-layer interface condition
}

\author{
Amirhossein Molavi Tabrizi, ${ }^{1}$ Spencer Goossens, ${ }^{1}$ Ali Mehdizadeh Rahimi, ${ }^{1}$ \\ Matthew Knepley, ${ }^{2}$ and Jaydeep P. Bardhan ${ }^{1}$ \\ ${ }^{1}$ Department of Mechanical and Industrial Engineering, Northeastern University, Boston, \\ Massachusetts 02115, USA \\ ${ }^{2}$ Department of Computational and Applied Mathematics, Rice University, Houston, Texas 77005, USA
}

(Received 2 November 2016; accepted 7 February 2017; published online 1 March 2017)

\begin{abstract}
We demonstrate that with two small modifications, the popular dielectric continuum model is capable of predicting, with high accuracy, ion solvation thermodynamics (Gibbs free energies, entropies, and heat capacities) in numerous polar solvents. We are also able to predict ion solvation free energies in water-co-solvent mixtures over available concentration series. The first modification to the classical dielectric Poisson model is a perturbation of the macroscopic dielectric-flux interface condition at the solute-solvent interface: we add a nonlinear function of the local electric field, giving what we have called a solvation-layer interface condition (SLIC). The second modification is including the microscopic interface potential (static potential) in our model. We show that the resulting model exhibits high accuracy without the need for fitting solute atom radii in a state-dependent fashion. Compared to experimental results in nine water-co-solvent mixtures, SLIC predicts transfer free energies to within $2.5 \mathrm{~kJ} / \mathrm{mol}$. The co-solvents include both protic and aprotic species, as well as biologically relevant denaturants such as urea and dimethylformamide. Furthermore, our results indicate that the interface potential is essential to reproduce entropies and heat capacities. These and previous tests of the SLIC model indicate that it is a promising dielectric continuum model for accurate predictions in a wide range of conditions. Published by AIP Publishing. [http://dx.doi.org/10.1063/1.4977037]
\end{abstract}

\section{INTRODUCTION}

Developing better models for thermodynamics of solutesolvent interactions is of crucial importance due to their wide range of applications in biology, nanotechnology, and fundamental chemistry. Implicit-solvent models play a variety of roles in these applications because their speed and simplicity make them appealing options where fully atomistic explicit-solvent models are impractical or impossible. ${ }^{1-3}$ Among the most popular implicit-solvent models are those based on statistical mechanical integral equations ${ }^{4-6}$ and those based on macroscopic dielectric theory and continuum electrostatics. ${ }^{1,7,8}$ The latter are widely used because they lead to well understood partial-differential equations for which a variety of numerical algorithms can be used to solve large problems. $^{9-15}$

However, the speed advantage of dielectric models comes at the cost of simplifying assumptions that make them unable to capture important phenomena. ${ }^{16-27}$ In particular, the most substantial errors are incurred in the continuum theory's treatment of the first layers of solvent molecules (the solvation layer) as a bulk dielectric material. Significant inaccuracies arise from the assumptions that solvent molecules (1) are infinitely small and (2) respond linearly with respect to an applied field. ${ }^{2,28}$ To remedy this shortcoming of classical continuum theories, different improvements have been suggested by researchers. To understand the behavior of solvent molecules in this layer, numerous groups have assessed physically motivated changes to solute atom radii $^{16,17}$ and conducted all-atom calculations with explicit solvent to probe solvationlayer response to a perturbing electric field. ${ }^{6,18,20,29-32}$ Another group used smooth Gaussian-based dielectric function to make a distinction between the solvent response at the boundary and the bulk response. ${ }^{33}$

These studies, which integrate extensive experimental and computational data, have supported the development of several dielectric-based models that address solvation-layer phenomena for water. ${ }^{17,34-40}$ Many focus on charge hydration asymmetry (CHA), that is, reproducing the fact that ions of equal size but opposite valence have different solvation free energies and entropies. Although existing models have provided improved treatment of CHA, they have generally treated all asymmetry as arising solely from water hydrogens approaching a solute more closely than the larger water oxygens. This phenomenon is known as steric asymmetry. In many continuum models, steric asymmetry is addressed using atom-type-specific or charge-dependent radii. ${ }^{16,17,34,38,41-44}$ Although effective radii do account for the effects of charge asymmetry for surface-exposed charges, the important point is that they cannot work for buried charges that are still within a few Angstroms of the surface: changing their radii cannot change the dielectric boundary. This suggests an alternative approach in which a physically meaningful, effective modification should be employed in the interface condition, rather than changing the interface directly (via the atom radii). ${ }^{24,26,27,45}$ The asymmetric response can be described as a combination of two distinct different mechanisms. ${ }^{24}$ The first one, as it was described above, is the steric asymmetry and the other one 
is the electrostatic interface potential that persists even if the solute is uncharged. ${ }^{18-20,46-48}$ This interface potential, which we call the static potential to distinguish it from the macroscopic notion, ${ }^{24}$ contributes substantially to solvation thermodynamics, though not to solvation free energies in the case of neutral solutes. In particular, the static-potential term contributes a term that is linear in the net charge, ${ }^{24,40}$ whereas the polarization contributes the familiar quadratic expression. For linear-response models, this quadratic dependence is clearly understood; for our nonlinear response model, it arises from the fact that the model responds linearly for virtually the entire charging process.

We have proposed a corrected dielectric continuum model that includes two simple modifications to treat these two solvation-layer phenomena directly and separately. ${ }^{24,26,27,49}$ First, the static potential is treated as a uniform field that does not change in response to the solute charge distribution. Second, we modify the familiar dielectric flux interface condition (obtained from macroscopic dielectric theory) by adding a nonlinear perturbation that depends on the local electric field. We call this the solvation-layer interface condition (SLIC) model, after the modified interface condition. ${ }^{45}$ Our initial work showed that SLIC accurately reproduces ion solvation free energies in water, as well as charge-hydration asymmetries on a challenging test set. ${ }^{26} \mathrm{We}$ then established that the widely used mean spherical approximation (MSA) in bulk solution theory ${ }^{50}$ could be approximated to give a SLIC-like nonlinear perturbation to the macroscopic dielectric interface condition. ${ }^{45}$ This work indicated that a temperature-dependent interface condition could accurately predict the solvation free energies and entropies in a variety of polar solvents. ${ }^{45}$ Most recently, SLIC has been extended for dilute electrolytes modeled with the linear Poisson-Boltzmann equation. ${ }^{27,49}$ This extended version was shown to accurately predict the charging free energies of individual atoms in polyatomic solutes. ${ }^{49}$ Remarkably, the model provides high accuracy without the need for parameterizing solute atom radii. The extra computational cost associated with using this model compared to the traditional continuum theory deserves discussion. Solving the nonlinear problem requires a sequence of standard continuum simulations. In the present work, spherical symmetry makes solutions very fast, and therefore the iterations continue until full convergence is achieved. For general solute molecules, we use five nonlinear Picard iterations, and so calculations are approximately five times more expensive. Note that the number of iterations can be reduced substantially in multiple ways, including using a Newton method rather than Picard; using more accurate initial guesses for the solution; and using multiresolution approaches.

In this paper, we test the SLIC model on two problems that are widely understood to challenge traditional dielectric continuum models. First, it is well known that such models fail to reproduce solvation thermodynamics; 51 the problem's importance has in fact motivated the parameterization of temperature-dependent radii. ${ }^{52}$ Second, relatively few implicit-solvent models have been applied to solvation in mixtures. ${ }^{53-56}$ Standard dielectric models have been shown to give poor accuracy in specific mixtures, ${ }^{57-59}$ but reference-interaction site model (RISM) theories ${ }^{60}$ and the continuum-based model COSMO-RS ${ }^{61-63}$ generally work well. One challenge for simple dielectric models is that correcting their oversimplifications, even in pure solvents, necessitates numerous correction terms with associated free parameters, making parameterization prohibitively complicated and time-consuming. For the studies here, where we use standard Shannon-Prewitt radii for the ions, ${ }^{43,64}$ SLIC has five fitting parameters. However, if parameterized to reproduce explicit-solvent simulations, the model has only three fitting parameters, which describe the nonlinear susceptibility in the solvation layer. ${ }^{26,27,49}$ Nevertheless, the model gives excellent results: the RMS error is $1.3 \mathrm{~kJ} / \mathrm{mol}$ for cations and $2.5 \mathrm{~kJ} / \mathrm{mol}$ for anions, in the 9 mixtures for which we have experimental data. Considering the model's simplicity, lack of chemical detail, and robustness to different solvents, this accuracy is surprising: it suggests that specific chemical interactions such as hydrogen bonds may not need to be explicitly included for predictive accuracy. This work addresses only monovalent ions because polyvalent ions induce dielectric saturation in the first shell, introducing an additional nonlinearity between the first and second shells. ${ }^{31,32,65-67}$ Ongoing work aims to extend SLIC to model polarization saturation around highly charged solutes.

The paper is organized as follows. The following section presents the SLIC model for the electrostatic component of molecular solvation free energies. Section III then addresses the application of SLIC to ion solvation thermodynamics in multiple polar solvents, and in Section IV we study ion solvation free energies in mixtures. Section V concludes the paper with a discussion of open questions, limitations, and areas for future work.

\section{THEORY}

Our model assumes that the solvation free energy can be decomposed as $\Delta G^{\text {solv }}=\Delta G^{n p}+\Delta G^{e s}$, where $\Delta G^{n p}$ represents the nonpolar free energy associated with growing a completely uncharged solute cavity into the solvent and $\Delta G^{e s}$ represents the free energy of creating the solute charge distribution. ${ }^{2}$ Because we are studying monovalent Born ions, we follow the typical convention and assume $\Delta G^{n p}$ is negligible, ${ }^{68}$ i.e., in this paper, we consider only the electrostatic solvation free energy and assume $\Delta G^{\text {solv }}=\Delta G^{e s}$. However, in other work, we have included a surface-area-dependent nonpolar term and optimized the nonpolar and electrostatic contributions simultaneously, obtaining good accuracy for small molecule solvation. ${ }^{49}$

In the standard (macroscopic) dielectric continuum model for $\Delta G^{e s}$, the solute is modeled as a dielectric medium with relative permittivity $\epsilon_{i n}$ that contains $N_{q}$ charges, usually at the atom centers (the $i$ th charge is $q_{i}$ and located at $\boldsymbol{r}_{i}$ ), and the solute potential satisfies the Poisson equation. The solvent exterior is modeled as an infinite homogeneous bulk dielectric with relative permittivity $\epsilon_{\text {out }}$, and in the absence of mobile charges (that is, in non-ionic solution), the solvent potential satisfies the Laplace equation. It is assumed that $\phi_{\text {out }} \rightarrow 0$ as $|\boldsymbol{r}| \rightarrow \infty$, and that the normal flux across the dielectric interface (denoted $S$ ) is given by the standard Maxwell interface 
condition

$$
\epsilon_{\text {in }} \frac{\partial \phi_{\text {in }}}{\partial n}\left(\boldsymbol{r}_{S_{-}}\right)=\epsilon_{\text {out }} \frac{\partial \phi_{\text {out }}}{\partial n}\left(\boldsymbol{r}_{S_{+}}\right),
$$

where $\partial / \partial n$ denotes the outward normal derivative, $\boldsymbol{r}_{S-}$ is a point just inside the dielectric boundary $S$, and $\boldsymbol{r}_{S+}$ is a point just outside. Solving this problem using finite difference methods ${ }^{69}$ or boundary integral methods, ${ }^{24,26,27,45}$ we obtain the reaction potential $\phi_{\text {reaction }}$ which arises due to the different permittivities. Overall, in the standard dielectric continuum model, we write the electrostatic component of the solvation free energy as $\Delta G^{e s}=\Delta G_{\text {reaction }}=\frac{1}{2} \sum_{i=1}^{N_{q}} q_{i} \phi_{\text {reaction }}\left(\boldsymbol{r}_{i}\right)$ where $\phi_{\text {reaction }}(\boldsymbol{r})$ is the reaction potential field.

In the SLIC model, by contrast, $\Delta G^{e s}$ is defined to be the sum of two terms

$$
\Delta G^{e s}=\Delta G_{\text {static }}+\Delta G_{\text {reaction }} .
$$

The first term in Eq. (2) captures the component of the charging free energy that arises due to the interfacial potential field $\phi_{\text {static }}(\boldsymbol{r})$ created by the solvent structure around a completely uncharged solute (i.e., an empty cavity with the solute shape). ${ }^{24,46}$ This term has been omitted in most previous dielectric continuum models, which leads to apparent deviations at very low charge densities. ${ }^{24,41,48}$ In this work, we assume the static potential field $\phi_{\text {static }}$ is constant everywhere inside the solute; validation and justification for this approximation can be found in Refs. 24, 26, and 46. The second term in Eq. (2) is the familiar polarization energy associated with solvent polarization in response to the solute charge distribution. However, in contrast to the standard dielectric model, we have replaced the dielectric interface condition of Eq. (1) with the solvation-layer interface condition $(\mathrm{SLIC})^{26,27}$

$$
\begin{aligned}
\left(\epsilon_{\text {in }}\right. & \left.-\Delta \epsilon h\left(E_{n}\left(\boldsymbol{r}_{S_{-}}\right)\right)\right) \frac{\partial \phi_{\text {in }}}{\partial n}\left(\boldsymbol{r}_{S_{-}}\right) \\
& =\left(\epsilon_{\text {out }}-\Delta \epsilon h\left(E_{n}\left(\boldsymbol{r}_{S_{-}}\right)\right)\right) \frac{\partial \phi_{\text {out }}}{\partial n}\left(\boldsymbol{r}_{S_{+}}\right)
\end{aligned}
$$

where $\Delta \epsilon=\epsilon_{\text {out }}-\epsilon_{\text {in }}$ and $E_{n}\left(\boldsymbol{r}_{S_{-}}\right)$is the normal electric field at $\boldsymbol{r}_{S_{-}}$(note that the electric field just outside the surface does not explicitly enter into the interface condition). Notice that this change makes the local dielectric contrast sensitive to the local electric field, and in particular changes the response to positive and negative fields (matching intuition about asymmetric solvation by water molecules). The perturbation $h\left(E_{n}\right)$ used here and in our previous work is

$$
h\left(E_{n}\right)=\alpha \tanh \left(\beta E_{n}-\gamma\right)+\mu .
$$

It has been shown ${ }^{26}$ that the suggested functional form is a suitable choice for our model and can capture the CHA effects. Figure 1 is a schematic plot of this perturbation. In this function, $\alpha$ dictates the magnitude of the deviation between suppressed response and enhanced response; $\beta$ determines the change in the electric field necessary to transition solvationlayer response between modes; $\gamma$ determines the critical electric field where the transition is centered; and $\mu$ determines where the suppressed response and enhanced response are situated with respect to the bulk response. It is important to note that the system responds linearly in regions where the derivative of $h$ is zero. Therefore, as $1 / \beta$, which corresponds to the

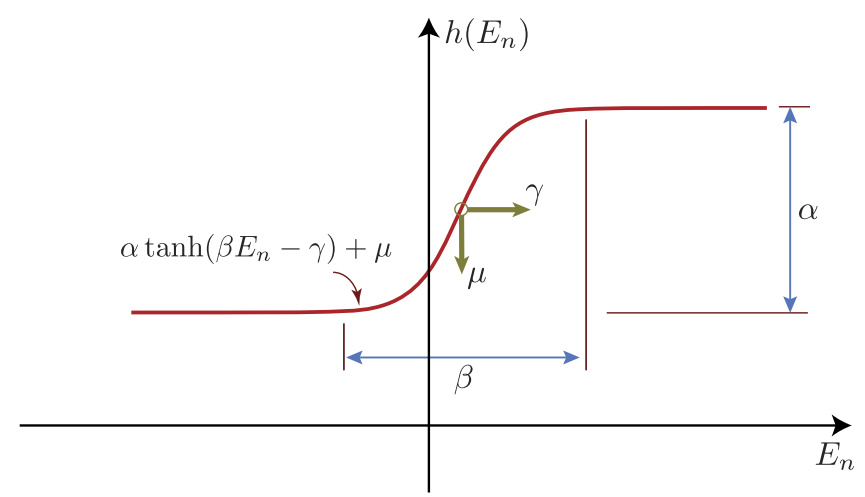

FIG. 1. Schematic of the SLIC perturbation to the standard dielectric interface condition and the different model parameters. In this function, $\alpha$ shows the magnitude of the deviation between the suppressed response and enhanced response; $1 / \beta$ determines the change in the electric field necessary to transition solvation-layer response between modes; $\gamma$ determines the critical electric field where the transition is centered; and $\mu$ dictates where the suppressed response and enhanced response are situated with respect to bulk response.

width of the transition, approaches zero, the system obeys two different regimes of linear response depending on the local field. ${ }^{24} \mathrm{~A}$ small but finite transition region allows the model to reproduce observed nonlinearities at low field strengths, which have been noted to arise due to the transition of solvent dipole orientations. ${ }^{70}$ However, for charged or highly polar compounds, this transition region's energetic contribution to solvation is quite small. ${ }^{45}$ In particular, because the actual region of nonlinear response happens in a very narrow region around $E_{n}=0$, the change in potential due to a change in solute charge is essentially linear for any finite charge. ${ }^{26,27,45}$ This gives essentially a piecewise-linear response model, and therefore the polarization component of the electrostatic solvation free energy can be approximated using the usual expression $\Delta G_{\text {reaction }}=\frac{1}{2} \sum q_{i} \phi_{\text {reaction }}\left(\boldsymbol{r}_{i}\right)$.

\section{PREDICTING SOLVATION THERMODYNAMICS}

To test whether the SLIC dielectric continuum model can reproduce solvation thermodynamics and to assess the effects of the static potential on prediction accuracy, we calculated ion solvation free energies, entropies, and heat capacities in nine polar solvents: water (abbreviated W), methanol $(\mathrm{MeOH})$, ethanol $(\mathrm{EtOH})$, formamide $(\mathrm{F})$, acetonitrile (AN), dimethylformamide (DMF), dimethyl sulfoxide (DMSO), nitromethane (NM), and propylene carbonate (PC). The test set was composed of the monovalent Born ions $\mathrm{Li}^{+}$, $\mathrm{Na}^{+}, \mathrm{K}^{+}, \mathrm{Rb}^{+}, \mathrm{Cs}^{+}, \mathrm{Cl}^{-}, \mathrm{Br}^{-}$, and $\mathrm{I}^{-}$; however, we could not use $\mathrm{Rb}^{+}$for $\mathrm{MeOH}, \mathrm{EtOH}, \mathrm{F}, \mathrm{DMSO}$, or NM due to a lack of experimental data. To parameterize the model and its temperature dependence, we used experimental solvation free energies at multiple temperatures; solvation free energy changes due to temperature were calculated using experimental solvation entropies and heat capacities. ${ }^{37}$ For each solvent/temperature pair, we parameterized the model once with $\phi_{\text {static }}$ set to zero and once with it allowed to vary. Note that ion radii were taken to be widely used values ${ }^{42}$ without any further adjustment. Thus, for each solvent/temperature parameterization, the fitting was overconstrained, having more data points ( 8 or 9 , see below) than model parameters ( 4 or 5 , depending on the use 
of $\left.\phi_{\text {static }}\right)$. Other relevant details for the solvents can be found in the supplementary material.

Figures 2 and 3 contain plots of SLIC predictions of ion solvation free energies and entropies at $25 \mathrm{C}$, along with predictions from standard Born theory and the asymmetric MSA theory ${ }^{42}$ for four solvents (W, $\mathrm{MeOH}, \mathrm{AN}$, and PC). The solvation free energies and entropy plots for the other five solvents are available in the supplementary material. Both SLIC models are substantially more accurate than the existing models. It is also clear that the SLIC model with the static potential is much more accurate than the one that omits it, especially for entropies (as well as free energies in F, AN, DMF, DMSO, NM, and PC). Interestingly, for anions, the SLIC model predicts exaggerated entropy differences compared to experiment; this is particularly noticeable for W, NM, and DMF. In addition, the cation entropies are generally more accurate than the anion entropies, but larger cations in AN are an exception. More detailed studies using explicit-solvent molecular dynamics are in progress.

Figure 4 contains plots of calculated heat capacities in W, $\mathrm{MeOH}, \mathrm{AN}$, and $\mathrm{PC}$ compared to the experimental data. Plots of the calculated heat capacities for the other solvents can be found in the supplementary material. Because heat capacities are related to the second derivative of the free energy, it is unsurprising that the correlations are weaker than for energies and entropies. As expected, the classical Born model is incapable of calculating heat capacities accurately. ${ }^{51}$ In our model, inaccuracies are particularly notable for anions, which may be related to their greater degree of charge transfer. ${ }^{71,72}$ The influence of the static potential on heat capacities is especially notable, providing an important offset to improve agreement with experiment in almost all cases. These results suggest that the static potential (an intrinsic property of the solvent and only weakly dependent on the shape of the uncharged solute) has a substantial effect on solutes' heat capacities, but that in these solvents, differences in heat capacities between molecules are governed by more detailed physics. In addition, we observe that small cations are problematic, which is not surprising because their high charge density leads to dielectric saturation, meaning that discrete solvent structure becomes increasingly important.

Together, Figures $2-4$ along with the corresponding figures in the supplementary material and the RMS errors presented in Table I indicate the importance of including the static potential in predicting solvation thermodynamics. The results also suggest that SLIC works well for solvents of various structure, complexity, and size, even though solvent structural details are not addressed explicitly. A table containing the values of each SLIC parameter at $T=25^{\circ} \mathrm{C}$, and their derivatives with respect to temperature, is available in the supplementary material. Previous work has shown that SLIC works well for polyatomic solutes such as biomolecules in water ${ }^{26}$ and future work will address such solutes in larger, more complex solvents such as PC. The present results do, however, explain previously noted questions, such as why $\Delta S$ does not have a straightforward dependence on ion radius, ${ }^{73}$ namely, the interface potential is largely independent of radius (though it does exhibit some variation ${ }^{18}$ ). Our model assumes $\phi_{\text {static }}$ is independent of solute shape, yet predicts these quantities
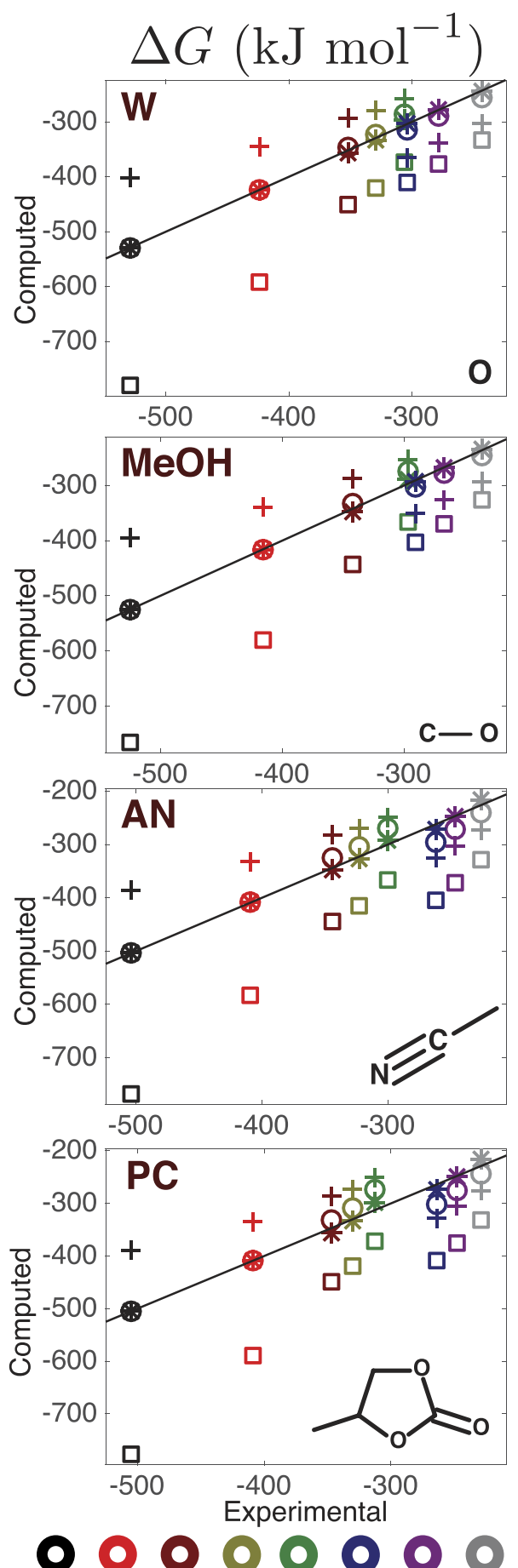

Li $\mathrm{Na} \mathrm{K} \quad \mathrm{Rb}$ Cs $\mathrm{Cl} \mathrm{Br}$ ।

\section{Classical Born}

+ Asymmetric MSA (Fawcett 1992)

SLIC without static potential

* SLIC with static potential

FIG. 2. Solvation free energies calculated by the classical Born dielectric model, the asymmetric MSA, ${ }^{42}$ and the SLIC model with and without the static potential. The solid lines represent perfect agreement between experiment and theory.

accurately, which suggests that its variation with size does not play a major role in ion solvation thermodynamics. We reiterate that the present model does not address second-shell 

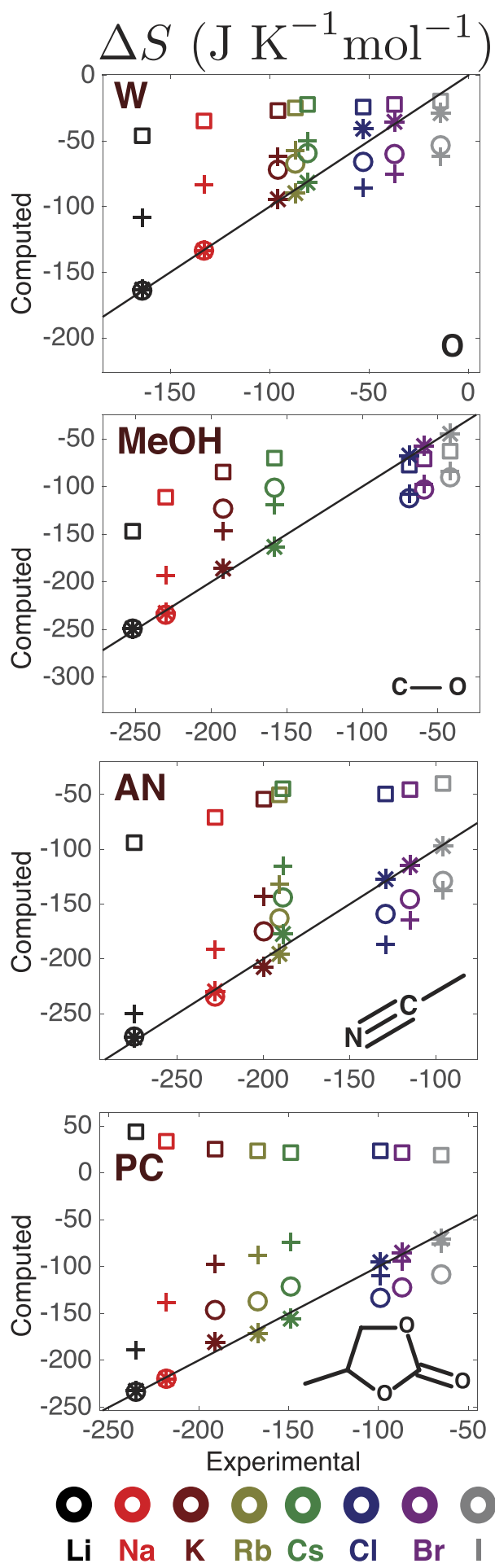

$\square$ Classical Born

+ Asymmetric MSA (Fawcett 1992)

SLIC without static potential * SLIC with static potential

FIG. 3. Entropies calculated by the classical Born dielectric model, the asymmetric MSA,${ }^{42}$ and the SLIC model with and without the static potential. The solid lines represent perfect agreement between experiment and theory.

effects, ${ }^{27}$ which is why the ions considered are only monovalent. Polyvalent ions that saturate the first shell ${ }^{17}$ will be studied in future work.
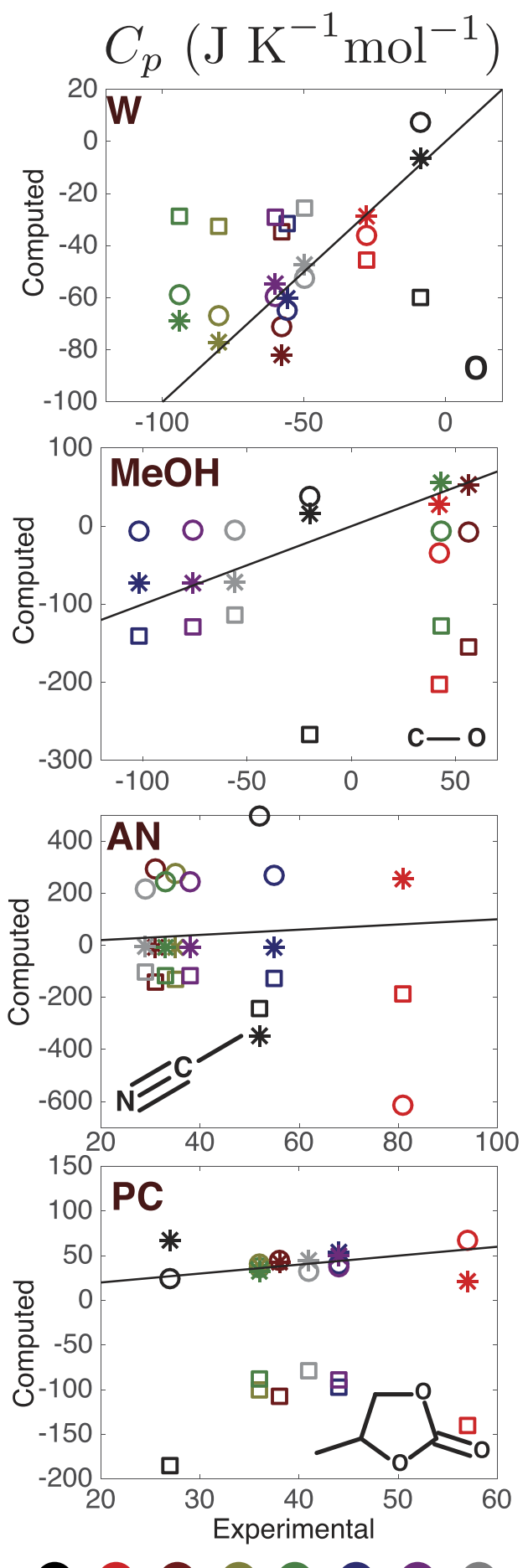

\section{- $\bigcirc-000$ \\ Li $\mathrm{Na} \mathrm{K}$ Rb Cs $\mathrm{Cl} \mathrm{Br}$ I \\ $\square$ Classical Born \\ O SLIC without static potential * SLIC with static potential}

FIG. 4. Heat capacities predicted by the classical Born theory and by the SLIC model with and without the static potential. The solid lines represent perfect agreement between experiment and theory.

Having established the SLIC models' accuracy, we next consider interpretation of the model parameters. Here we study only the model that includes the static potential $\phi_{\text {static }}$ contribution because significant data argue for its inclusion and the 
TABLE I. RMS errors, for solvation free energies $\Delta G$, entropies $\Delta S$, and heat capacities $C p$, calculated by SLIC with and without static potential.

\begin{tabular}{|c|c|c|c|c|c|c|}
\hline \multirow[b]{2}{*}{ Solvent } & \multicolumn{2}{|c|}{$\Delta G\left(\mathrm{~kJ} \mathrm{~mol}^{-1}\right)$} & \multicolumn{2}{|c|}{$\Delta S\left(\mathrm{~J} \mathrm{~mol}^{-1}\right)$} & \multicolumn{2}{|c|}{$C_{p}\left(\mathrm{~J} \mathrm{~K}^{-1} \mathrm{~mol}^{-1}\right)$} \\
\hline & Without $\phi_{\text {static }}$ & With $\phi_{\text {static }}$ & Without $\phi_{\text {static }}$ & With $\phi_{\text {static }}$ & Without $\phi_{\text {static }}$ & With $\phi_{\text {static }}$ \\
\hline $\mathrm{W}$ & 10.45 & 4.04 & 21.38 & 6.86 & 15.83 & 12.59 \\
\hline $\mathrm{MeOH}$ & 11.50 & 3.64 & 44.89 & 3.63 & 68.02 & 19.71 \\
\hline AN & 21.45 & 6.06 & 28.34 & 5.35 & 348.88 & 159.48 \\
\hline PC & 24.26 & 8.23 & 31.58 & 5.29 & 6.06 & 19.59 \\
\hline
\end{tabular}

model without it exhibits significantly poorer accuracy. Also, because we used standard Shannon and Prewitt radii rather than radii based on any MD simulations, the optimized parameter values cannot be interpreted directly in terms of atomistic molecular simulations. That is, explicit-solvent simulations and a SLIC model based on MD radii are needed to provide a consistent model comparison and offer atomistic insights into the model's treatment of solvation-layer response. However, it is worth noting common features of the temperature-dependent response. For all solvents, the width of the transition region in Figure 1 (as captured by $1 / \beta$ ) is increasing with temperature, which can be interpreted in terms of increased thermal motion leading to more gradual transition, as a function of the local electric field. Results for $\alpha(T)$, the magnitude of the response asymmetry (between enhanced and suppressed response) are not consistent: $\mathrm{W}, \mathrm{MeOH}, \mathrm{AN}, \mathrm{NM}$, and PC exhibit increases in response asymmetry with temperature (positive $\frac{\partial \alpha}{\partial T}$ ), while the others exhibit decreasing trends. The centering parameters $\mu$ and $\gamma$ increase with temperature for all solvents, but the significance of this is not clear. The static potentials for all solvents are negative, and increasing (becoming less negative) with temperature, but more detailed simulation will be required to establish the relationship to microscopic phenomena.

TABLE II. Prediction of Gibbs free energy, entropy, and heat capacity in the model with $\phi_{\text {static }}$. Values in parentheses are experimental values when available.

\begin{tabular}{lcccc}
\hline \hline Solvent & Ion & $\Delta G\left(\mathrm{~kJ} \mathrm{~mol}^{-1}\right)$ & $\Delta S\left(\mathrm{~J} \mathrm{~K}^{-1} \mathrm{~mol}^{-1}\right)$ & $C_{p}\left(\mathrm{~J} \mathrm{~K}^{-1} \mathrm{~mol}^{-1}\right)$ \\
\hline $\mathrm{W}$ & $\mathrm{F}^{-}$ & $-430(-429)^{43}$ & $-67(-115)^{43}$ & $-86(-45)^{75}$ \\
\hline $\mathrm{MeOH}$ & $\mathrm{Rb}^{+}$ & $-326(-319)$ & $-178(-175)$ & 55 \\
& $\mathrm{~F}^{-}$ & -415 & -116 & $-79(-131)^{75}$ \\
\hline EtOH & $\mathrm{Rb}^{+}$ & $-319(-313)$ & $-197(-187)$ & 128 \\
& $\mathrm{~F}^{-}$ & -405 & -145 & $-153(-194)$ \\
\hline $\mathrm{F}$ & $\mathrm{Rb}^{+}$ & $-340(-334)$ & $-135(-130)$ & 27 \\
& $\mathrm{~F}^{-}$ & -418 & -128 & $36(28)^{76}$ \\
\hline AN & $\mathrm{F}^{-}$ & -390 & -192 & 147 \\
\hline DMF & $\mathrm{F}^{-}$ & -389 & -230 & 105 \\
\hline DMSO & $\mathrm{Rb}^{+}$ & $-348(-339)$ & $-151(-180)$ & 32 \\
& $\mathrm{~F}^{-}$ & -400 & -160 & $186(60)^{75}$ \\
\hline NM & $\mathrm{Rb}^{+}$ & $-324(-318)$ & $-186(-183)$ & 19 \\
\hline PC & $\mathrm{F}^{-}$ & -391 & -182 & $95(71)^{75}$ \\
\hline \hline & $\mathrm{F}^{-}$ & -394 & -149 & 67 \\
\hline
\end{tabular}

We may also consider these solvents from the perspective of being protic or aprotic. The protic solvents water, $\mathrm{MeOH}$, and EtOH exhibit positive correlations between dielectric constant and $\alpha$ and $\beta$ but negative correlations between the dielectric constant and $\gamma$ and $\mu$. However, formamide does not follow this trend. For aprotic solvents, the parameters do not exhibit any obvious dependency on the dielectric constant or the solvent radius (supplementary material). Future work will address these relationships in more detail, and particularly focus on the extent to which solvation entropies and heat capacities are in fact related to the parameters' temperature dependence.

Table II contains calculations for solvation thermodynamics where the experimental data are not yet available or were not used in parameterization. These cases include $\mathrm{F}^{-}$in all solvents, and $\mathrm{Rb}^{+}$in $\mathrm{MeOH}, \mathrm{EtOH}, \mathrm{F}, \mathrm{DMSO}$, and NM. Because solvation of fluoride ions in nonaqueous solvents has received limited attention, ${ }^{74}$ we did not use this ion for parameterizing SLIC in any of the solvents, even in water, where it has been studied. ${ }^{43,75}$ Table II also includes available experimental measurements. Again, the model predicts free energies and entropies accurately and is qualitatively reasonable for heat capacities (especially compared to other models).

\section{PREDICTING SOLVATION IN MIXTURES}

We parameterized concentration-dependent SLIC models for ion solvation in 9 water-co-solvent mixtures. The cosolvents were acetone (AC), acetonitrile (AN), dioxane (Diox), dimethyl ether (DME), dimethylformamide (DMF), dimethyl sulfoxide (DMSO), ethanol (EtOH), methanol $(\mathrm{MeOH})$, and urea. We obtained ion solvation free energies in each mixture by adding tabulated transfer free energies ${ }^{77-85}$ to experimental ion solvation free energies in water. ${ }^{43}$ Mixture dielectric constants were taken to be experimental values. ${ }^{86-93}$ The experimental transfer free energies included the monovalent Born ions $\mathrm{Li}^{+}, \mathrm{Na}^{+}, \mathrm{K}^{+}, \mathrm{Rb}^{+}, \mathrm{Cs}^{+}, \mathrm{Cl}^{-}, \mathrm{Br}^{-}$, and $\mathrm{I}^{-}$, though transfer free energies were not available for every ion in every co-solvent. Each SLIC parameter was modeled as varying quadratically (for example, $\alpha(c)=\alpha_{0}+\alpha_{1} c+\alpha_{2} c^{2}$ ) where the co-solvent weight/weight concentration $c$ between 0 , meaning pure water, and a maximum of 1 , pure co-solvent. However, transfer free energies from pure water to pure co-solvent were not available. Thus, for each solvent, the 5 SLIC dependent parameters led to a fitting of 15 parameters over all experimental data associated with that co-solvent mixture, regardless of concentration. For each optimization, every solvation free 
energy was weighted equally in the optimization problem, and every co-solvent had at least 36 measured transfer energies. Therefore, each optimization problem was well posed. Again, no ion radii were fit during this work: the Shannon-Prewitt radii were used unchanged. ${ }^{43,64}$ The optimization problems were unconstrained, and for initial guesses we used coefficients obtained by polynomial fitting from parameterizations at individual mixture concentrations. We verified the model consistency by using the optimized SLIC models of different mixtures to predict solvation free energies in neat water (supplementary material). MATLAB's non-linear least squares function was used for optimization (version 2015b on Mac).

Table III contains the root-mean-square (RMS) errors for the SLIC model associated with each co-solvent, tabulated separately for cations and anions. Errors are given for both the absolute solvation free energies and for the transfer free energies from neat water to a given mixture. The model achieves high accuracy, with RMS errors for solvation free energies less than $7 \mathrm{~kJ} / \mathrm{mol}$. However, differences can be observed, particularly in that the cation predictions are somewhat less accurate than predictions for anions. Transfer free energies (measured as the solvation free energy difference between the model at $0 \%$ co-solvent and the model at finite co-solvent weight fraction) are highly accurate, with both cations and anions achieving RMS errors of less than $2.5 \mathrm{~kJ} / \mathrm{mol}$, though cation transfer free energies are more accurate than those for anions.

Figures 5 and 6 are plots of the cation transfer free energies and anion transfer free energies, respectively, into mixtures of water and DMSO; these are the representative of the results for all solvents. The supplementary material contains individual plots for the transfer free energy profile for each Born ion in each co-solvent mixture, compared to both experiment and the prediction of continuum Born theory. For the Born model, we held the Shannon-Prewitt radii fixed but changed the dielectric constant according to experiment. The cation transfer free energy profiles are well reproduced in our theory; cesium, the largest, is underpredicted by a small but consistent amount. For anions, the experimental profiles exhibit a wide variance as the concentration increases (Figure 6); these results are observable to a lesser extent for other mixtures, including ethanol, DMF, and dioxane. With regard to relative transfer free energies between cations, our model reproduces experimental orderings reasonably well over the concentration range for

TABLE III. RMS errors, in $\mathrm{kJ} / \mathrm{mol}$, for $\Delta G^{\text {solv }}$ and $\Delta G^{t r}$, computed separately for cations and anions.

\begin{tabular}{lccccc}
\hline \hline & \multicolumn{2}{c}{$\Delta G^{\text {solv }}$} & & \multicolumn{2}{c}{$\Delta G^{\text {tr }}$} \\
\cline { 2 - 3 } \cline { 6 - 6 } Solvent & Cations & Anions & & Cations & Anions \\
\hline AC & 6.64 & 1.10 & & 0.13 & 1.38 \\
AN & 2.25 & 1.27 & & 0.86 & 0.95 \\
Diox & 2.71 & 1.95 & & 1.09 & 1.81 \\
DME & 5.07 & 1.14 & & 0.42 & 1.50 \\
DMF & 4.62 & 0.75 & & 1.29 & 1.07 \\
DMSO & 2.22 & 2.58 & & 0.47 & 1.71 \\
EtOH & 4.45 & 2.41 & & 0.86 & 2.46 \\
MeOH & 2.24 & 1.50 & & 0.41 & 0.54 \\
Urea & 2.48 & 1.63 & 0.72 & 0.53 \\
\hline \hline
\end{tabular}

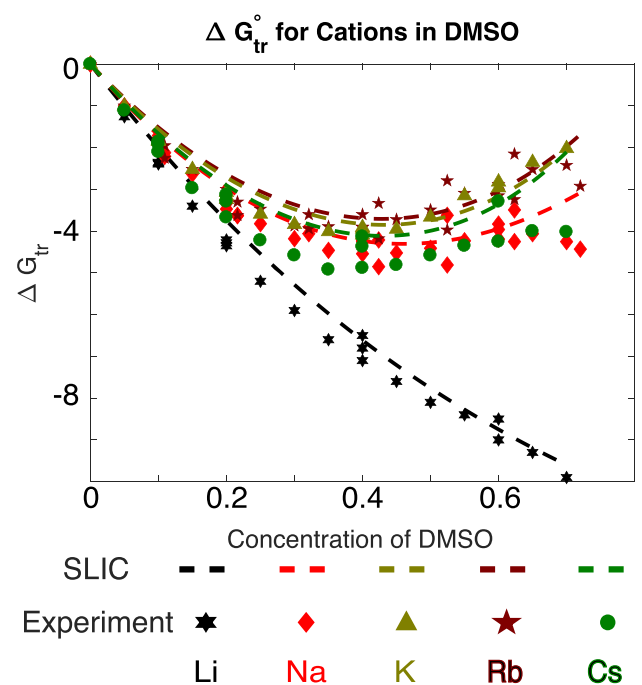

FIG. 5. Transfer free energies, in $\mathrm{kJ} / \mathrm{mol}$, for cations into water-DMSO mixtures.

which experiments are available, with the exception of potassium. For anions, however, the SLIC differences are underpredicted compared to the experimental measurements. The complete set of transfer free energy profiles are available in the supplementary material, offering additional evidence of the SLIC model's accuracy. First, in DMSO as well as in ethanol, urea, DMF, DME, and dioxane, SLIC reproduces cations' concave-up transfer free energy profiles and concave-down profiles for anions. Furthermore, in water-methanol mixtures, the cation profiles are concave down and the anions concave up, and SLIC reproduces this difference (although predictions for potassium exhibit poorer accuracy). Second, for acetone and acetonitrile, the anion transfer free energies vary essentially linearly with concentration, which our model also reproduces. Third, transfer free energies for cations in ethanol mixtures exhibit an inflection point, and our model reproduces the overall profiles accurately, though not the change in curvature.

We also show in the supplementary material that with fixed radii, the classical Born model, which uses purely macroscopic

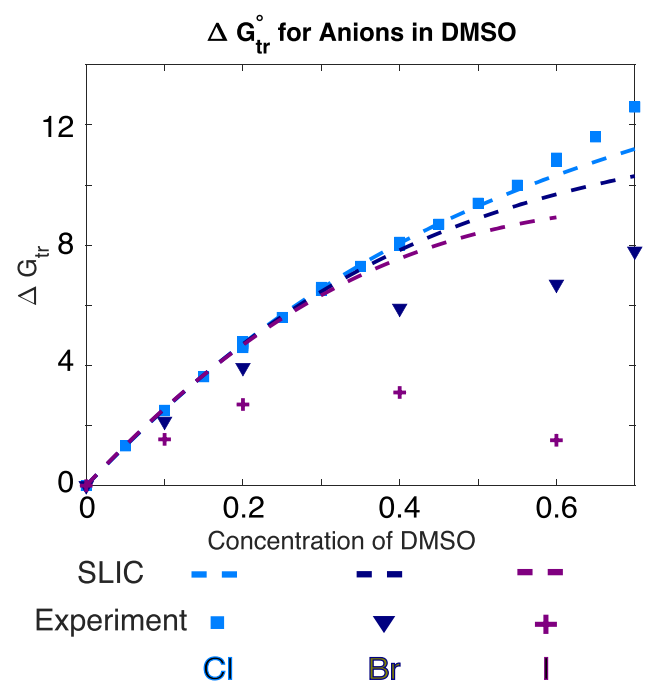

FIG. 6. Transfer free energies, in $\mathrm{kJ} / \mathrm{mol}$, for anions into water-DMSO mixtures. 
dielectric notions, is unable to reproduce even qualitative features because the only varying parameter is the dielectric constant. For example, the transfer free energy profiles often have significant curvature and a local maximum or minimum, whereas the Born-model profiles are monotonic (see particularly the results for dioxane). To construct an accurate Born model, each ion's radius must be parameterized at each cosolvent concentration; one observes a non-monotonic variation in radius that can be as large as $0.1 \AA$ (supplementary material). As a blind prediction to test the SLIC model, the supplementary material also includes predictions for ion transfer free energies in co-solvent mixtures for which we did not find any reference data. These predictions included fluorine for all cosolvents, as well as lithium and sodium in acetone and ethanol. For water-ethanol mixtures, the lithium and sodium transfer free energy profiles are very similar to the other cations' profiles. In contrast, the predictions for acetone are quite different for larger cations, which suggests that such experiments or atomistic simulations would offer a stringent test of our model.

\section{DISCUSSION}

We have established that a dielectric continuum solvent model can accurately reproduce ion solvation thermodynamics in a variety of polar solvents and solvation free energies in mixtures, provided that (1) the usual macroscopic dielectric interface condition is replaced with a solvation-layer interface condition (SLIC), and (2) proper account is taken for the interface potential, which we have termed a static potential in order to highlight its microscopic character. ${ }^{46}$ Importantly, the SLIC model achieves this accuracy via a simple physical picture rather than a chemical one-that is, SLIC does not account explicitly for solvent chemical properties such as hydrogen bonding propensities or detailed solvent structure. Instead, the model treats in essence the re-orientation response of asymmetric dipoles in the solvation layer. Overall, however, SLIC works very well for both protic and aprotic solvents, as well as for solvents of varying size and structure; other work has established its accuracy for predicting solvation free energies of polyatomic solutes. ${ }^{26,27}$ Our assessment of transfer free energies in mixtures offers encouraging signs for the model's robustness. For mixtures, our results were obtained via global optimization (over all concentrations for a given co-solvent) with parameters varying quadratically as a function of concentration. However, even linear dependencies work reasonably well for most solvents, despite the reduced number of fitting parameters (supplementary material). Furthermore, SLIC models implemented to match explicit-solvent MD need even fewer parameters. ${ }^{26}$ SLIC predicts, with semiquantitative accuracy, the experimental free energies of transfer over a wide range of concentrations, even when the dependencies have different trends over the Born ions. The accuracy and generality suggest that first-shell solvent response, as captured via a surface-charge representation, suffices to explain a large component of changes in solvation over substantial changes in solvent composition. To put our present model to a stringent test, we have predicted solvation thermodynamics and transfer free energies for cases in which we have no experimental data (primarily fluorine, as well as lithium and sodium; see the supplementary material). Our calculations of entropies and heat capacities also indicate the importance of separating the static potential from the nonlinear polarization response.

In work on polyatomic solutes including amino acids, we have shown that the model does not require atom radii to be adjusted for atomic charge, ${ }^{26}$ which differs from numerous suggestions and parameterizations. The present work shows that the SLIC continuum model is highly accurate even when the solute atom radii are independent of solvent composition and temperature. ${ }^{41,52}$ In contrast to the models which parameterize many radii (making model comparison challenging), in the SLIC model, we have instead changed the interface condition and introduced the static potential. In our view, this is a more meaningful adjustment because the system changes involve the solvent and the solvent-solute interactions, not the solute. We note that this viewpoint is implicit in the MSA model for Born ion solvation. ${ }^{43}$ Our model's rather surprising accuracy provides further support that temperature-dependent changes in the average charge structure of the solvation layer, rather than specific chemical interactions, are responsible for ion solvation thermodynamics. We have predicted solvation entropies with high accuracy and heat capacities with only reasonable accuracy, but this lower accuracy is not surprising given that heat capacities are second-derivative quantities, and more chemical detail is likely to be needed for these predictions.

For mixtures, straightforward calculations illustrate a clear weakness of classical dielectric models: Born radii fit to experimental results must vary non-monotonically with cosolvent concentration. This firmly establishes the notion that in traditional Poisson models, the atom radii must be considered as free (adjustable) parameters. ${ }^{94}$ In contrast, all of our calculations here used the standard Shannon-Prewitt radii; ${ }^{64}$ when SLIC is parameterized against explicit-solvent MD simulations, the resulting model is accurate using standard MD Lennard-Jones radii with only a uniform scaling. ${ }^{26,49}$ In this respect, our model has dozens of fewer fitting parameters than traditional continuum electrostatic models, where radii must be fit for each atom type or for many groups of similar type. It is worth emphasizing that recent models of chargehydration asymmetry are similarly able to reproduce wide sets of experimental data using fewer radii fitting parameters 35,38 than classical continuum models.

A question of significant interest is how to reconcile the solvation-layer response picture of SLIC with the significant literature on the role of solvent fluctuations, ${ }^{95-98}$ which our model does not include. For example, can the solvent fluctuation density field be decomposed into terms related to the static and reaction fields? It is also interesting to consider the relationship of our approach to the local molecular field (LMF) theory of Weeks et al., which approximates the exact Yvon-Born-Green hierarchy. ${ }^{6,99}$ Both models determine the electrostatic potential field inside the solute and could be compared in fine detail. In ongoing work, we are assessing SLIC's capacity to predict the stabilities of cation-anion contact pairs in solution and the impact of including SLIC in the polarizable continuum model (PCM). ${ }^{100,101}$ Compared to the existing implicit-solvent models for mixtures, SLIC differs in four 
primary ways. First, numerous models have been proposed for specific co-solvents, but to our knowledge only COSMORS and RISM-based models have been demonstrated on the large number of co-solvents as we have shown here. We have also shown that our model reproduces experimental trends in transfer free energies with high accuracy, that is, our model captures dependencies on concentration, in addition to being accurate at specific co-solvent concentrations. Third, SLIC has already been shown to work very well for polyatomic solutes with complex geometries; in contrast, many existing models have focused only on spherical ions or spherical nanoparticles. The exceptions here again are RISM-based models and COSMO-RS. Fourth, SLIC represents a remarkably small modification of traditional Poisson-Boltzmann based dielectric models, and can be incorporated easily into the large number of finite-difference, finite-element, or boundary-element solvers. ${ }^{27,49}$

The model's simplicity comes with attendant limitations and open questions, and the tests presented here cover only a fraction of possible applications. Results on mixtures suggest that accuracy tends to decrease at high co-solvent concentrations. These deviations were surprising given the model's accuracy for the neat co-solvents. Unfortunately, the neat cosolvent solvation free energies were inconsistent with the transfer free energies available to us, precluding their use as data points at $100 \%$ concentration. We hope that future experimental measurements or explicit-solvent simulations may provide insights into these errors. Ongoing work aims to predict the solvation of polyatomic solutes in mixtures and to investigate whether SLIC can predict molecular solvation thermodynamics in mixtures as it can in neat solvents. We have also not yet tested the model on mixtures of polar and non-polar solvents. There exist several implicit-solvent models for such mixtures, ${ }^{54,55,102}$ and whether SLIC works for these solutions is not known. Other current work extends our analysis here to a SLIC variant that can model dissolved ions in the solvent mixture using the linearized Poisson-Boltzmann equation. ${ }^{49}$ This requires an additional nonlinear interface condition at the Stern (ion-exclusion) surface, and in mixtures the width of this ion-exclusion region will presumably depend on the co-solvent size and concentration. We have distinguished the static potential field from the macroscopic notion of an interface potential. Because it arises from mean solvent structure around a solute, the field satisfies the Poisson equation; however, steric considerations mean that the static potential near the boundary is not actually uniform in a thin region at the surface of the solute (the first layer of solute atoms). ${ }^{26,46}$ Future work will investigate whether biological systems exploit this non-uniformity for molecular function, which may necessitate the development of a more sophisticated static potential model than the present assumption of a uniform field. Future work will also investigate whether the functional form of the solvation-layer correction may be better fit to an error function than the present hyperbolic tangent. ${ }^{103}$ One additional open question is whether SLIC can be applied to understand protein behavior in mixtures of water and osmolytes or denaturants.

Our development of SLIC originally only focused on solutes in water and arose from a question that included theoretical, philosophical, and practical considerations: What would an accurate implicit solvent model look like if one did not specifically parameterize atom radii but simply used the values employed in MD? That is, if one insisted on using atom radii that were not parameterized individually, would it even be possible to modify the standard dielectric continuum model in such a way as to obtain accurate charging free energies? It has been claimed that atom radii must of necessity be parameterized based on the atom type and given charge, and it is certainly true that the accuracy of standard dielectric models can be extremely high in specific geometries when using radii that have been parameterized individually. As we have discussed, our view is to ask how well one can reproduce the electrostatic contribution to the formally exact PMF obtained by integrating out all solvent degrees of freedom. Given that an accurate model should reproduce an entire charging free energy curve, not only the final charging free energy, we investigated the properties of standard dielectric models in reproducing these charging free energy profiles and found them poor. This finding was, of course, in total agreement with the common wisdom that radii should be charge dependent. Even more importantly, however, we found that the charging free energies for individual charges were nearly quadraticimplying that linear response does hold, but with a small but crucial change in curvature depending on whether the charge increases from zero and becomes positive, or whether it decreases from zero and becomes negative. ${ }^{24}$ The standard dielectric continuum model, with its much larger number of parameters, achieves high accuracy in important cases that represent a deceptively incomplete assessment of the free energy landscape as a function of charge (that is, the final, total charging free energy). The standard dielectric model errs further in that its omission of the static potential means that there are small deviations from linear response at low charging densities; for a clear example, see the widely used, and highly accurate, continuum radii optimized by Roux et al. ${ }^{41}$ These deviations are not dielectric saturation in the traditional macroscopic sense of high-electric-field maximal polarization, but work done to overcome the "structuring force" induced by water structure around the fully uncharged solute (see, for example, Ref. 46). Traditional dielectric models err by subsuming the static potential contribution (which is linear in the solute charge) into the reaction potential response. Further study is warranted, but we estimate that detailed parameterization of atomic radii should correct for this, for small molecules. For large molecules, the effects are more subtle, depending on the size of the molecule and the relative distribution of charged groups to the surface or the interior.

To illustrate the importance of treating the two effects (steric asymmetry and the static potential) separately, we have recently computed the charging free energies (and profiles) of individual atoms in small molecules. ${ }^{49}$ The static potential derived from MD simulations in TIP3P water is positive, meaning that for small positive charges, the charging free energy is actually positive (unfavorable). Traditional dielectric models are completely unable to reproduce such positive charging free energies, regardless of atomic charge or radius. However, our model reproduces these energies with quantitative accuracy, and we note that these represent unfavorable electrostatic contributions to solvation by hydrophobic groups. 
Traditional implicit-solvent models predicate that hydrophobic groups contribute nothing; for instance, nonpolar solvation models are often parameterized under the assumption that alkanes have electrostatic solvation free energies equal to zero. We have found this to be not accurate. Furthermore, because our model has few parameters that do not involve the atom radii, we have been able to parameterize a complete and consistent implicit-solvent model-that is, parameterizing both the electrostatic and nonpolar terms simultaneously, such that our nonpolar model does not need to also correct for the charging free energy of hydrophobic compounds. ${ }^{49}$ We found that the complete SLIC implicit-solvent model is remarkably accurate, comparable to explicit solvent simulations for solvation free energies and water-octanol transfer free energies.

Speaking more generally, we were motivated theoretically by curiosity about why an implicit-solvent model should need to use different atom radii depending on the sign of its charge. For a monoatomic ion of a given chemical radius, its reaction potential can always be written in terms of an appropriate surface charge on a sphere of that radius, but the surface charge density might depend on the sign (and magnitude) of the charge. Philosophically, it seemed reasonable to consider that the solute atom did not change physically when embedded in a solvent of different composition or temperature, so the use of a state-dependent radius seemed like a way to correct deeper problems with the dielectric theory. Practically, our development of SLIC arose from a simple motivation: the desire to avoid the need for extensive parameterization of radii with every new solvent theory. A number of more proper justifications may be offered as well. First, there is an increasing interest from environmental and biotechnological research in the prediction of protein function at different temperatures. Second, both basic and applied biosciences research focuses on the effects of changing solution conditions such as the addition of co-solvents, or partition coefficients for transfer free energies between neat solvents. ${ }^{104,105}$ Third, the costs and complexity of continuum-model parameterization and validation seem to be limiting the community's ability to use continuum models to address the massive chemical diversity associated with post-translational modifications of proteins. Fourth, there exist already a wide range of continuum solvers based on the PB theory, including large-scale parallel codes, ${ }^{106,107}$ codes coupled to $\mathrm{MD},{ }^{108}$ and many in quantum chemistry. ${ }^{100}$ The model's successes in this work and other recent studies motivate adapting some of these software packages for more challenging tests of the SLIC model.

\section{SUPPLEMENTARY MATERIAL}

The MATLAB source code for the solvation thermodynamics calculations can be accessed at: https://bitbucket.org/ bardhanlab/slic_solvation_thermodynamics. The MATLAB source code for the solvent mixture calculations can be accessed at https://bitbucket.org/bardhanlab/si-slic-mixtures. Supplementary material for the thermodynamics calculations includes solvent details, SLIC parameters at $T=25^{\circ} \mathrm{C}$ and their derivatives with respect to temperature, the full set of plots for solvation free energies, entropies, and heat capacities in all neat polar solvents, and the associated RMS errors. Supplementary material for the mixture calculations include (1) plots of all transfer free energy profiles for Born ions in all 9 co-solvent mixtures, compared to experiment and the classical Born model, under three types of parameterized SLIC models: quadratic concentration-dependence (discussed in this paper), a model with linear concentrationdependence (fewer fitting parameters), and a model with quadratic concentration-dependence where missing experimental data has been supplanted with interpolated results from polynomial fits to experiment; (2) validation of the co-solvent model's consistency by calculation of solvation free energies in neat water (i.e., at $0 \%$ co-solvent); (3) RMS errors for the cations and anions in different co-solvent mixtures, for the three types of parameterized SLIC models; (4) concentrationdependent Born radii required to fit experimental ion solvation free energies in water-ethanol mixtures.

\section{ACKNOWLEDGMENTS}

The authors thank F. C. Pickard IV, B. Brooks, M. Reuter, A. E. Ismail, P. Jungwirth, D. Green, N. Baker, and E. Boyden for valuable discussions. MGK was partially supported by the U.S. Department of Energy, Office of Science, Advanced Scientific Computing Research, under Contract No. DE-AC02-06CH11357, and the National Science Foundation under Award Nos. SI2-SSI 1450339 and OCI-1147680. The work of J.P.B., S.G., and A.M.R. has been supported in part by the National Institute of General Medical Sciences (NIGMS) of the National Institutes of Health (NIH) under Award No. R21GM102642 and by a contract with Battelle. The content is solely the responsibility of the authors and does not necessarily represent the official views of the National Institutes of Health.

${ }^{1}$ K. A. Sharp and B. Honig, "Electrostatic interactions in macromolecules: Theory and applications," Annu. Rev. Biophys. Biophys. Chem. 19, 301332 (1990).

${ }^{2}$ B. Roux and T. Simonson, "Implicit solvent models," Biophys. Chem. 78, 1-20 (1999).

${ }^{3}$ L. Li, L. Wang, and E. Alexov, "On the energy components governing molecular recognition in the framework of continuum approaches," Front. Mol. Biosci. 2, 5 (2015).

${ }^{4}$ A. Kovalenko and F. Hirata, "Three-dimensional density profiles of water in contact with a solute of arbitrary shape: A RISM approach," Chem. Phys. Lett. 290, 237-244 (1998).

${ }^{5}$ T. Luchko, S. Gusarov, D. R. Roe, C. Simmerling, D. A. Case, J. Tuszynski, and A. Kovalenko, "Three-dimensional molecular theory of solvation coupled with molecular dynamics in Amber," J. Chem. Theory Comput. 6, 607-624 (2010).

${ }^{6}$ R. C. Remsing and J. D. Weeks, “The role of local response in ion solvation: Born theory and beyond," J. Phys. Chem. B 120, 6238 (2016).

${ }^{7}$ J. G. Kirkwood, "Theory of solutions of molecules containing widely separated charges with special application to zwitterions," J. Chem. Phys. 2, 351 (1934).

${ }^{8}$ J. Tomasi and M. Persico, "Molecular interactions in solution: An overview of methods based on continuous descriptions of the solvent," Chem. Rev. 94, 2027-2094 (1994).

${ }^{9}$ M. K. Gilson, A. Rashin, R. Fine, and B. Honig, "On the calculation of electrostatic interactions in proteins,” J. Mol. Biol. 184, 503-516 (1985).

${ }^{10}$ B. H. Honig, W. L. Hubbell, and R. F. Flewelling, "Electrostatic interactions in membranes and proteins," Ann. Rev. Biophys. Biophys. Chem. 15, 163193 (1986). 
${ }^{11}$ M. K. Gilson and B. Honig, "Calculation of the total electrostatic energy of a macromolecular system: Solvation energies, binding energies, and conformational analysis," Proteins: Struct., Funct., Genet. 4, 7-18 (1988).

${ }^{12}$ B. Honig and A. Nicholls, "Classical electrostatics in biology and chemistry," Science 268, 1144-1149 (1995).

${ }^{13}$ H. Fan, A. E. Mark, J. Zhu, and B. Honig, "Comparative study of generalized born models: Protein dynamics," Proc. Natl. Acad. Sci. U. S. A. 102(19), 6760-6764 (2005).

${ }^{14}$ J. Carlsson, M. Nervall, M. Andér, and J. Åqvist, "Continuum solvation models in the linear interaction energy method," J. Phys. Chem. B 110, 12034-12041 (2006)

${ }^{15}$ C. Bertonati, B. Honig, and E. Alexov, "Poisson-Boltzmann calculations of nonspecific salt effects on protein-protein binding free energies," Biophys. J. 92, 1891-1899 (2007).

${ }^{16}$ W. M. Latimer, K. S. Pitzer, and C. M. Slansky, "The free energy of hydration of gaseous ions, and the absolute potential of the normal calomel electrode," J. Chem. Phys. 7, 108-112 (1939).

${ }^{17}$ A. A. Rashin and B. Honig, "Reevaluation of the Born model of ion hydration,” J. Phys. Chem. 89(26), 5588-5593 (1985).

${ }^{18} \mathrm{H}$. S. Ashbaugh, "Convergence of molecular and macroscopic continuum descriptions of ion hydration," J. Phys. Chem. B 104(31), 7235-7238 (2000).

${ }^{19}$ R. M. Lynden-Bell, J. C. Rasaiah, and J. P. Noworyta, "Using simulation to study solvation in water," Pure Appl. Chem. 73, 1721-1731 (2001).

${ }^{20}$ S. Rajamani, T. Ghosh, and S. Garde, "Size dependent ion hydration, its asymmetry, and convergence to macroscopic behavior," J. Chem. Phys. 120, 4457 (2004)

${ }^{21}$ M. V. Fedorov and A. A. Kornyshev, "Unravelling the solvent response to neutral and charged solutes," Mol. Phys. 105, 1-16 (2007).

${ }^{22}$ D. L. Mobley, A. E. Barber, II, C. J. Fennell, and K. A. Dill, "Charge asymmetries in hydration of polar solutes," Phys. Chem. B 112, 2405-2414 (2008).

${ }^{23}$ C. J. Fennell and K. A. Dill, "Physical modeling of aqueous solvation," J. Stat. Phys. 145, 209 (2011).

${ }^{24}$ J. P. Bardhan, P. Jungwirth, and L. Makowski, "Affine-response model of molecular solvation of ions: Accurate predictions of asymmetric charging free energies," J. Chem. Phys. 137, 124101 (2012).

${ }^{25}$ Y. Shi and T. L. Beck, "Length scales and interfacial potentials in ion hydration," J. Chem. Phys. 139, 044504 (2013).

${ }^{26}$ J. P. Bardhan and M. G. Knepley, "Modeling charge-sign asymmetric solvation free energies with nonlinear boundary conditions," J. Chem. Phys. 141, 131103 (2014).

${ }^{27}$ J. P. Bardhan, D. Tejani, N. Wieckowski, A. Ramaswamy, and M. G. Knepley, "A nonlinear boundary condition for continuum models of biomolecular electrostatics," in Progress in Electromagnetics Research Symposium (PIERS), 2015.

${ }^{28}$ D. Beglov and B. Roux, "Solvation of complex molecules in a polar liquid: An integral equation theory," J. Chem. Phys. 104(21), 8678-8689 (1996).

${ }^{29}$ A. Grossfield, "Dependence of ion hydration on the sign of the ion's charge," J. Chem. Phys. 122, 024506 (2005).

${ }^{30}$ G. Hummer, A. E. García, and D. M. Soumpasis, "A statistical mechanical description of biomolecular hydration," Faraday Discuss. 103, 175-189 (1996).

${ }^{31}$ B. Jayaram, R. Fine, K. Sharp, and B. Honig, "Free energy calculations of ion hydration: An analysis of the born model in terms of microscopic simulations," J. Phys. Chem. 93, 4320-4327 (1989).

${ }^{32}$ H. E. Alper and R. M. Levy, "Field strength dependence of dielectric saturation in liquid water," J. Phys. Chem. 94, 8401-8403 (1990).

${ }^{33} \mathrm{~L}$. Li, C. Li, Z. Zhang, and E. Alexov, "On the dielectric 'constant' of proteins: Smooth dielectric function for macromolecular modeling and its implementation in delphi," J. Chem. Theory Comput. 9, 2126-2136 (2013).

${ }^{34}$ E. O. Purisima and T. Sulea, "Restoring charge asymmetry in continuum electrostatic calculations of hydration free energies," J. Phys. Chem. B 113, 8206-8209 (2009).

${ }^{35}$ C. R. Corbeil, T. Sulea, and E. O. Purisima, "Rapid prediction of solvation free energy. 2. The first-shell hydration (FiSH) continuum model," J. Chem. Theory Comput. 6, 1622-1637 (2010).

${ }^{36}$ W. R. Fawcett and L. Blum, "The role of dipole-dipole interactions in the solvation of monoatomic monovalent ions in water on the basis of the mean spherical approximation," J. Electroanal. Chem. 355, 253-263 (1993).

${ }^{37}$ A. C. Chamberlin, C. J. Cramer, and D. G. Truhlar, "Extension of a temperature-dependent aqueous solvation model to compounds containing nitrogen, fluorine, chlorine, bromine, and sulfur," J. Phys. Chem. B 112, 3024-3039 (2008).
${ }^{38}$ A. Mukhopadhyay, B. H. Aguilar, I. S. Tolokh, and A. V. Onufriev, "Introducing charge hydration asymmetry into the generalized Born model," J. Chem. Theory Comput. 10, 1788-1794 (2014).

${ }^{39}$ T. T. Duignan, D. F. Parsons, and B. W. Ninham, "Collins's rule, Hofmeister effects and ionic dispersion interactions," Chem. Phys. Lett. 608, 55-59 (2014).

${ }^{40}$ M. M. Reif and P. H. Hünenberger, "Origin of asymmetric solvation effects for ions in water and organic solvents investigated using molecular dynamics simulations: The Swain acity-basity scale revisited," J. Phys. Chem. B 120, 8485 (2016).

${ }^{41}$ M. Nina, D. Beglov, and B. Roux, "Atomic radii for continuum electrostatics calculations based on molecular dynamics free energy simulations," J. Phys. Chem. B 101, 5239-5248 (1997).

${ }^{42}$ L. Blum and W. R. Fawcett, "Application of the mean spherical approximation to describe the Gibbs solvation energies of monovalent monoatomic ions in polar solvents," J. Phys. Chem. 96(1), 408-414 (1992).

${ }^{43}$ W. R. Fawcett, Liquids, Solutions, and Interfaces: From Classical Macroscopic Descriptions to Modern Microscopic Details (Oxford University Press, New York, 2004).

${ }^{44}$ R. Sundararaman and W. A. Goddard III, "The charge asymmetric nonlocally determined local-electric solvation model," J. Chem. Phys. 142, 064107 (2015).

${ }^{45}$ A. Molavi Tabrizi, M. Knepley, and J. P. Bardhan, "Generalizing the mean spherical approximation as a multiscale, nonlinear boundary condition at the solute-solvent interface," Mol. Phys. 114, 2558-2567 (2016).

${ }^{46}$ D. S. Cerutti, N. A. Baker, and J. A. McCammon, "Solvent reaction field potential inside an uncharged globular protein: A bridge between implicit and explicit solvent models?," J. Chem. Phys. 127, 155101 (2007).

${ }^{47}$ S. M. Kathmann, I.-F. W. Kuo, C. J. Mundy, and G. K. Schenter, "Understanding the surface potential of water," J. Phys. Chem. B 115, 4369-4377 (2011).

${ }^{48}$ Y.-L. Lin, A. Aleksandrov, T. Simonson, and B. Roux, "An overview of electrostatic free energy comptutations for solutions and proteins," J. Chem. Theory Comput. 10, 2690-2709 (2014).

${ }^{49}$ A. Molavi Tabrizi, S. Goossens, C. D. Cooper, M. G. Knepley, and J. P. Bardhan, "Extending the solvation-layer interface condition (SLIC) continuum electrostatic model to linearized Poisson-Boltzmann solvent," J. Chem. Theory Comput. (submitted).

${ }^{50}$ L. Blum and D. Q. Wei, "Analytical solution of the mean spherical approximation for an arbitrary mixture of ions in a dipolar solvent," J. Chem. Phys. 87(1), 555-565 (1987).

${ }^{51}$ P. Vath, M. B. Zimmt, D. V. Matyushov, and G. A. Voth, "A failure of continuum theory: Temperature dependence of the solvent reorganization energy of electron transfer in highly polar solvents," J. Phys. Chem. B 103(43), 9130-9140 (1999).

${ }^{52}$ A. H. Elcock and J. A. McCammon, "Continuum solvation model for studying protein hydration thermodynamics at high temperatures," J. Phys. Chem. B 101(46), 9624-9634 (1997).

${ }^{53}$ E. C. Allen and G. C. Rutledge, "Evaluating the transferability of coarsegrained, density-dependent implicit solvent models to mixtures and chains," J. Chem. Phys. 130, 034904 (2009).

${ }^{54}$ M. Basilevsky, A. Odinkov, E. Nikitina, F. Grigoriev, N. Petrov, and M. Alfimov, "Preferential solvation of spherical ions in binary DMSO/benzene mixtures," J. Chem. Phys. 130, 024504 (2009).

${ }^{55}$ A. Bonincontro, C. Cametti, B. Nardiello, S. Marchetti, and G. Onori, "Dielectric behavior of DNA in water-organic co-solvent mixtures," Biophys. Chem. 121, 7-13 (2006).

${ }^{56}$ I. Nakamura, A. Shi, and Z. Wang, "Ion solvation in liquid mixtures: Effects of solvent reorganization," Phys. Rev. Lett. 109, 257802 (2012).

${ }^{57}$ W. Jarzeba, G. C. Walker, A. E. Johnson, and P. F. Barbara, "Nonexponential solvation dynamics of simple liquids and mixtures," Chem. Phys. 152, 5768 (1999).

${ }^{58} \mathrm{O}$. W. Kolling, "Dielectric continuum assumption as a description of medium effects in binary solvent mixtures: Implications for electrontransfer processes in cosolvent systems," J. Phys. Chem. 95, 3950-3954 (1991).

${ }^{59}$ T. A. Barnes, J. W. Kaminski, O. Borodin, and T. F. Miller III, “Ab initio characterization of the electrochemical stability and solvation properties of condensed-phase ethylene carbonate and dimethyl carbonate mixtures," J. Phys. Chem. C 119, 3865-3880 (2015).

${ }^{60}$ K. Yoshida, T. Yamaguchi, A. Kovalenko, and F. Hirata, "Structure of tertbutyl alcohol-water mixtures studied by the RISM theory," J. Phys. Chem. B 106, 5042-5049 (2002). 
${ }^{61}$ A. Klamt, F. Eckert, and M. Hornig, "COSMO-RS: A novel view to physiological solvation and partition questions," J. Comput.-Aided Mol. Des. 15, 355-365 (2001).

${ }^{62} \mathrm{~A}$. Klamt, "The COSMO and COSMO-RS solvation models," Wiley Interdiscip. Rev.: Comput. Mol. Sci. 1, 699-709 (2011).

${ }^{63} \mathrm{~A}$. Klamt and M. Diedenhofen, "Calculation of solvation free energies with DCOSMO-RS," J. Phys. Chem. A 119, 5439-5445 (2015).

${ }^{64}$ R. D. Shannon and C. T. Prewitt, "Effective ionic radii in oxides and fluorides," Acta Crystallogr., Sect. B: Struct. Crystallogr. Cryst. Chem. 25, 925-946 (1969).

${ }^{65}$ L. Sandberg, R. Casemyr, and O. Edholm, "Calculated hydration free energies of small organic molecules using a nonlinear dielectric continuum model," J. Phys. Chem. B 106, 7889-7897 (2002).

${ }^{66}$ A. A. Kornyshev and G. Sutmann, "Nonlocal dielectric saturation in liquid water," Phys. Rev. Lett. 79, 3435-3438 (1997).

${ }^{67}$ Y. Marcus, "Thermodynamics of solvation of ions. Part 5. Gibbs free energy of hydration at 298.15 K," J. Chem. Soc. Faraday Trans. 87, 2995-2999 (1991).

${ }^{68}$ E. O. Purisima, "Fast summation boundary element method for calculating solvation free energies of macromolecules," J. Comput. Chem. 19(13), 1494-1504 (1998).

${ }^{69}$ W. Rocchia, S. Sridharan, A. Nicholls, E. Alexov, A. Chiabrera, and B. Honig, "Rapid grid-based construction of the molecular surface and the use of induced surface charge to calculate reaction field energies: Applications to the molecular systems and geometric objects," J. Comput. Chem. 23, 128-137 (2002).

${ }^{70}$ S. W. Rick and B. J. Berne, "The aqueous solvation of water: A comparison of continuum methods with molecular dynamics," J. Am. Chem. Soc. 116, 3949-3954 (1994).

${ }^{71}$ Z. Zhao, D. M. Rogers, and T. L. Beck, "Polarization and charge transfer in the hydration of chloride ions," J. Chem. Phys. 132, 014502 (2010).

${ }^{72}$ D. M. Rogers and T. L. Beck, "Quasichemical and structural analysis of polarizable anion hydration,” J. Chem. Phys. 132, 014505 (2010).

${ }^{73} \mathrm{M}$. Salomon, "Thermodynamics of ion solvation in water and propylene carbonate," J. Phys. Chem. 74, 2519-2524 (1970).

${ }^{74}$ G. T. Hefter, "Solvation of fluoride ions. 3. A review of fluoride solvation thermodynamics in nonaqueous and mixed solvents," Rev. Inorg. Chem. 10, 185-224 (1989).

${ }^{75}$ Y. Marcus and G. Hefter, "Ionic partial molar heat capacities in non-aqueous solvents," J. Chem. Soc. Faraday Trans. 92, 757-761 (1996).

${ }^{76}$ T. Chen, G. Hefter, R. Buchner, and G. Senanayake, "Molar volumes and heat capacities of electrolytes and ions in nonaqueous solvents: 1 . Formamide," J. Solution Chem. 27, 1067-1096 (1998).

${ }^{77}$ C. F. Wells, "Ionic solvation in methanol + water mixtures: Free energies of transfer from water," J. Chem. Soc. Faraday Trans. 69, 984-992 (1973).

${ }^{78}$ C. F. Wells, "Ionic solvation in water + co-solvent mixtures. Part 2. Free energies of transfer of single ions from water into mixtures of water with acetone, isopropanol, glycerol or methanol," J. Chem. Soc. Faraday Trans. 70, 694-704 (1953).

${ }^{79}$ C. F. Wells, "Ionic solvation in water + co-solvent mixtures. Part 9. Free energies of transfer of single ions from water into water + ethanol mixtures," J. Chem. Soc. Faraday Trans. 80, 2445-2458 (1984).

${ }^{80}$ C. F. Wells, "Ionic solvation in water + co-solvent mixtures. Part 7. Free energies of transfer of single ions from water into water + dimethylsulphoxide mixtures," J. Chem. Soc. Faraday Trans. 77, 1515-1528 (1981).

${ }^{81}$ G. S. Groves and C. F. Wells, "Ionic solvation in water + co-solvent mixtures. Part 10. Free energies of transfer of single ions from water into water + ethanonitrile mixtures," J. Chem. Soc. Faraday Trans. 81, 1985-1997 (1985).

${ }^{82}$ G. S. Groves and C. F. Wells, "Ionic solvation in water + co-solvent mixtures. Part 11. Free energies of transfer of single ions from water into water + urea mixtures," J. Chem. Soc. Faraday Trans. 81, 3091-3102 (1985).

${ }^{83}$ C. F. Wells, "Ionic solvation in water + co-solvent mixtures. Part 24. Free energies of transfer of single ions from water into water $+1,2-$ dimethoxyethane mixtures," Thermochim. Acta 208, 323-339 (1992).

${ }^{84}$ C. F. Wells, "Ionic solvation in water + co-solvent mixtures. Part 6 . Free energies of transfer of single ions from water into water + dioxan mixtures," J. Chem. Soc. Faraday Trans. 74, 1569-1582 (1978).
${ }^{85}$ I. M. Sidahmed and C. F. Wells, "Ionic solvation in water + co-solvent mixtures. Part 15. Free energies of transfer of single ions from water into water + dimethylformamide mixtures," J. Chem. Soc. Faraday Trans. 84(4), 1153-1162 (1988).

${ }^{86} \mathrm{~S}$. J. Suresh and V. M. Naik, "Theory of dielectric constant of aqueous solutions," J. Chem. Phys. 116, 4212-4220 (2002).

${ }^{87}$ S. A. Markarian and L. S. Gabrielyan, "Dielectric relaxation study of diethylsulfoxide/water mixtures," Phys. Chem. Liq. 47, 311-321 (2009).

${ }^{88} \mathrm{G}$. Akerlof, "Dielectric constants of some organic solvent-water mixtures at various temperatures," J. Am. Chem. Soc. 54, 4125-4139 (1932).

${ }^{89}$ K. I. Roy and C. A. Lucy, "Dielectric friction as a mechanism for selectivity alteration in capillary electrophoresis using acetonitrile-water media," Electrophoresis 23, 383-392 (2002).

${ }^{90}$ J. Wyman, Jr., "Dielectric constants: Ethanol-diethyl ether and urea-water solutions between $0^{\circ}$ and $50^{\circ}$," J. Am. Chem. Soc.55(10):4116-4121 (1933).

${ }^{91}$ G. Douhéret and A. Pal, "Dielectric constants and densities of aqueous mixtures of 2-alkoxyethanols at $25^{\circ} \mathrm{C}, "$ J. Chem. Eng. Data 33, 40-43 (1988).

${ }^{92} \mathrm{G}$. Akerlof and O. A. Short, "The dielectric constants of dioxane-water mixtures between $0^{\circ}$ and $80^{\circ}$," J. Am. Chem. Soc. 58, 1241-1243 (1936).

${ }^{93}$ A. C. Kumbharkhane, S. M. Puranik, and S. C. Mehrotra, "Dielectric relaxation studies of aqueous n, n-dimethylformamide using a picosecond time domain technique," J. Solution Chem. 22, 219-229 (1993).

${ }^{94} \mathrm{~A}$. Papazyan and A. Warshel, "A stringent test of the cavity concept in continuum dielectrics," J. Chem. Phys. 107, 7975-7978 (1997).

${ }^{95}$ G. Hummer, L. R. Pratt, and A. E. Garcia, "Hydration free energy of water," J. Phys. Chem. 99, 14188-14194 (1995).

${ }^{96}$ G. Hummer, L. R. Pratt, and A. E. García, "Multistate Gaussian model for electrostatic solvation free energies," J. Am. Chem. Soc. 119, 8523-8527 (1997).

${ }^{97}$ D. R. Martin and D. V. Matyushov, "Electrostatic fluctuations in cavities within polar liquids and thermodynamics of polar solvation," Phys. Rev. E 78, 041206 (2008).

${ }^{98}$ S. Sarupria and S. Garde, "Quantifying water density fluctuations and compressibility of hydration shells of hydrophobic solutes and proteins," Phys. Rev. Lett. 103, 037803 (2009).

${ }^{99}$ J. M. Rodgers and J. D. Weeks, "Local molecular field theory for the treatment of electrostatics," J. Phys.: Condens. Matter 20, 494206 (2008).

${ }^{100}$ S. Miertus, E. Scrocco, and J. Tomasi, "Electrostatic interactions of a solute with a continuum - A direct utilization of $a b$ initio molecular potentials for the prevision of solvent effects," Chem. Phys. 55(1), 117-129 (1981).

${ }^{101}$ B. Mennucci, "Continuum solvation models: What else can we learn from them?," J. Phys. Chem. Lett. 1, 1666-1674 (2010).

${ }^{102}$ M. Basilevsky, A. Odinkov, E. Nikitina, and N. Petrov, "The dielectric continuum solvent model adapted for treating preferential solvation effects," $J$. Electroanal. Chem. 660, 339-346 (2011).

${ }^{103}$ S. W. Sides, G. S. Grest, and M.-D. Lacasse, "Capillary waves at liquid-vapor interfaces: A molecular dynamics simulation,” Phys. Rev. E 60, 6708-6713 (1999).

${ }^{104}$ F. Helmer, K. Kiehs, and C. Hansch, "The linear free-energy relation between partition coefficients and the binding, and conformational perturbation of macromolecules by small organic compounds," Biochemistry $\mathbf{7}$, 2858-2863 (1968).

${ }^{105}$ C. A. Lipinski, F. Lombardo, B. W. Dominy, and P. J. Feeney, "Experimental and computational approaches to estimate solubility and permeability in drug discovery and development settings," Adv. Drug Delivery Rev. 23, 3-25 (1997).

${ }^{106}$ N. A. Baker, D. Sept, M. J. Holst, and J. A. McCammon, "Electrostatics of nanoysystems: Application to microtubules and the ribosome," Proc. Natl. Acad. Sci. U. S. A. 98, 10037-10041 (2001).

${ }^{107} \mathrm{C}$. Li, M. Petukh, L. Li, and E. Alexov, "Continuous development of schemes for parallel computing of the electrostatics in biological systems: Implementation in DelPhi," J. Comput. Chem. 34, 1949-1960 (2013).

${ }^{108}$ Q. Cai, M.-J. Hsieh, J. Wang, and R. Luo, "Performance of nonlinear finitedifference Poisson-Boltzmann solvers," J. Chem. Theory Comput. 6, 203211 (2010). 